\title{
Growth of $\mathrm{ZnO}$ nanotetrapods with hexagonal crown
}

\author{
Fa-Quan He and Ya-Pu Zhao ${ }^{\text {a) }}$ \\ State Key Laboratory of Nonlinear Mechanics (LNM), Institute of Mechanics, Chinese Academy of Sciences, \\ Beijing 100080, People's Republic of China
}

(Received 30 November 2005; accepted 9 March 2006; published online 10 May 2006)

\begin{abstract}
$\mathrm{ZnO}$ nanotetrapods with hexagonal crown were synthesized on a silicon wafer by vapor transport process at a low temperature of $630{ }^{\circ} \mathrm{C}$ and normal pressure without the presence of catalysts. The results demonstrated that the as-synthesized products with slender legs and regular hexagonal crown are single crystal with wurtzite structure and preferentially grow up along (001) direction. Photoluminescence spectra revealed that the green emission originated from oxygen vacancies overwhelmed that of the near-band-edge ultraviolet peak, which suggests the peculiar-shaped nanotetrapods may have potential applications in multichannel nano-optoelectronic devices. (C) 2006 American Institute of Physics. [DOI: 10.1063/1.2202003]
\end{abstract}

Recently, one-dimensional (1D) or quasi-1D $\mathrm{ZnO}$ nanostructured materials have received special attention due to their unique properties and numerous potential applications. High chemical stability, low threshold intensity, wide band gap $(3.37 \mathrm{eV})$, and large exciton binding energy (60 meV) make $\mathrm{ZnO}$ an excellent candidate for the fabrication of electronic and optoelectronic nanodevices. In order to obtain different $\mathrm{ZnO}$ nanostructured materials, varieties of methods have been developed. By far, a large number of $\mathrm{ZnO}$ nanostructured materials with different structures and morphologies have been synthesized, such as nanobelts, ${ }^{1}$ nanowires, ${ }^{2}$ nanospring, ${ }^{3}$ nanorods, ${ }^{4,5}$ nanocombs, ${ }^{6}$ tetrapodlike nanostructures, ${ }^{7}$ nanotubes, ${ }^{8}$ nanonails, ${ }^{9}$ nanosheets, ${ }^{10}$ and nanohelices, ${ }^{11}$ their characteristics and potential applications have been studied intensively.

Considering the quantum confinement effect, peculiar properties of $\mathrm{ZnO}$ nanostructure may be expected to be obtained through controlling the shape and dimensionality of the materials. The nanotetrapods structured materials with special shape and structure which are significantly different from other $\mathrm{ZnO}$ nanostructures, have remarkable optical, electric, magnetic, and mechanical properties, and they possess promising applications in nanoelectronics and photonics. Thus, much effort has been devoted to the synthesis and investigation of their characteristics.

In this letter, we report a simple way to synthesize the special nanotetrapods. Compared with other nanotetarpods structured materials, the as-synthesized products have much larger surface area. The sample exhibits extraordinary strong green emissions that are related with the oxygen vacancies. This special characteristic suggests that the nanotetrapod material possesses high electrical conductivity, which indicates that they may be used for electron nanoconductors in multichannel optoelectronic devices. ${ }^{12}$

The special $\mathrm{ZnO}$ nanotetrapods were synthesized via vapor evaporation deposition at a low temperature. A quartz tube was mounted horizontally inside a high-temperature tube furnace (with $50 \mathrm{~mm}$ inner diameter and $1200 \mathrm{~mm}$ in length). A $p$-type silicon (111) wafer (pretreated by $\mathrm{H}_{2} \mathrm{SO}_{4}$ : $\mathrm{H}_{2} \mathrm{O}_{2}=7: 3$ for $30 \mathrm{~min}$, then washed by de-ionized water and

\footnotetext{
a) Author to whom correspondence should be addressed; electronic mail: yzhao@lnm.imech.ac.cn
}

ethanol three times, respectively, and finally dried naturally) was placed face down on an alumina ceramic boat (10 $\times 70 \mathrm{~mm}^{2}$ ) loaded with $1 \mathrm{~g}$ zinc powders (purity: $99.999 \%$ ) and the vertical distance between the substrate and the zinc source was about $6 \mathrm{~mm}$. The boat was inserted into the center of the horizontal quartz tube. Different from other methods, neither catalyst nor vacuum was needed in our experiment. For the sake of driving off the $\mathrm{O}_{2}$ in the tube, an $\mathrm{Ar}$ flow with a rate of 1000 SCCM (SCCM denotes cubic centimeter per minute at STP) was turned on and kept for about $20 \mathrm{~min}$ and then closed before heating. Subsequently, the quartz tube was heated at a rate of $30{ }^{\circ} \mathrm{C} / \mathrm{min}$. When the temperature reached $630{ }^{\circ} \mathrm{C}$, a flow of Ar at a rate of about 300 SCCM and $\mathrm{O}_{2}$ at about 20 SCCM was switched on and kept for 10-30 min. Finally, the heating power and the flow of $\mathrm{Ar}$ and $\mathrm{O}_{2}$ were turned off. After cooling down to room temperature, white material was found on the surface of the substrates.

The as-made nanostructured $\mathrm{ZnO}$ were examined using $\mathrm{X}$-ray diffraction (XRD, D/MAX-2500), field emission scanning electron microscopy (FE-SEM, FEI SIRION), highresolution field emission transmission electron microscopy (HR-FETEM, JEM 2010F), Raman spectrum (JY-T64000, excitation wavelength: $524 \mathrm{~nm}$ ), and the photoluminescence (PL) property $(900 \mathrm{nF}, \mathrm{Xe}$ lamp, $325 \mathrm{~nm}$, as the excitation source).

The XRD analysis was employed to determine the structure and phase of the products. Figure 1 illustrates the XRD pattern of the as-prepared products. All the peaks are indexed to typical wurtzite hexagonal phase of $\mathrm{ZnO}$ with lattice constants of $a=3.250 \AA$ and $c=5.206 \AA$. No peaks for $\mathrm{Zn}$ or other impurities were detected in the spectrum indicating that the as-grown samples are pure $\mathrm{ZnO}$. Furthermore, it also implies that the nanostructured $\mathrm{ZnO}$ is highly crystallized.

The morphology and structure of the prepared products were analyzed by SEM. Figure 2(a) shows the typical SEM image and reveals that the products obtained are uniform tetrapodlike $\mathrm{ZnO}$ with regular hexagonal crown on each leg. In a perfect $\mathrm{ZnO}$ nanotetrapod unit, four nanopods share a common crystal facet and each leg is a uniform and regular hexagonal crown. The typical TEM image provides further information of the microstructure of the synthesized nanotetrapods with hexagonal crown. Figures 2(b) and 2(c) show that the four legs of a unit are uniform, the diameter of the 


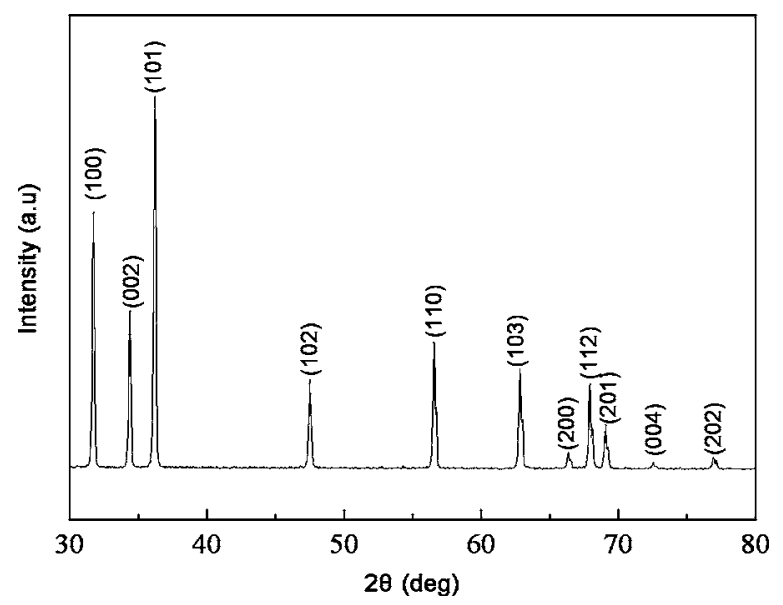

FIG. 1. XRD pattern of $\mathrm{ZnO}$ nanotetrapods with hexagonal crown.

four legs ranges from 50 to $100 \mathrm{~nm}$ and their lengths are between $1-1.5 \mu \mathrm{m}$. The side length of hexagonal crown is about $500 \mathrm{~nm}$ and around $50 \mathrm{~nm}$ in thick, respectively. The detailed information of the $\mathrm{ZnO}$ nanotetrapod was studied by HR-FETEM equipped with selected area electron diffraction (SAED). The SAED pattern as shown in Fig. 2(f) suggests that they are single crystals with wurtzite structure, which is in accord with the XRD results. The HR-TEM image [Fig. $2(\mathrm{e})]$ of the crystal corroborates that the synthesized $\mathrm{ZnO}$ are perfect crystalline and grown along the (001) direction.

The growth mechanism was thought to be vapor solid (VS) rather than conventionally vapor-liquid-solid (VLS) model because the synthesis was processed without any catalysts or other additives. The configuration of the grown crystal reflects its internal structure, and the growth rate of different face determines its own morphology. Generally speaking, the crystallography is dependent on the symmetry of the external structure, the bonding manner between the structural unit and the crystal defects, but the external factors play an important role in it. From Fig. 3(a), we can observe
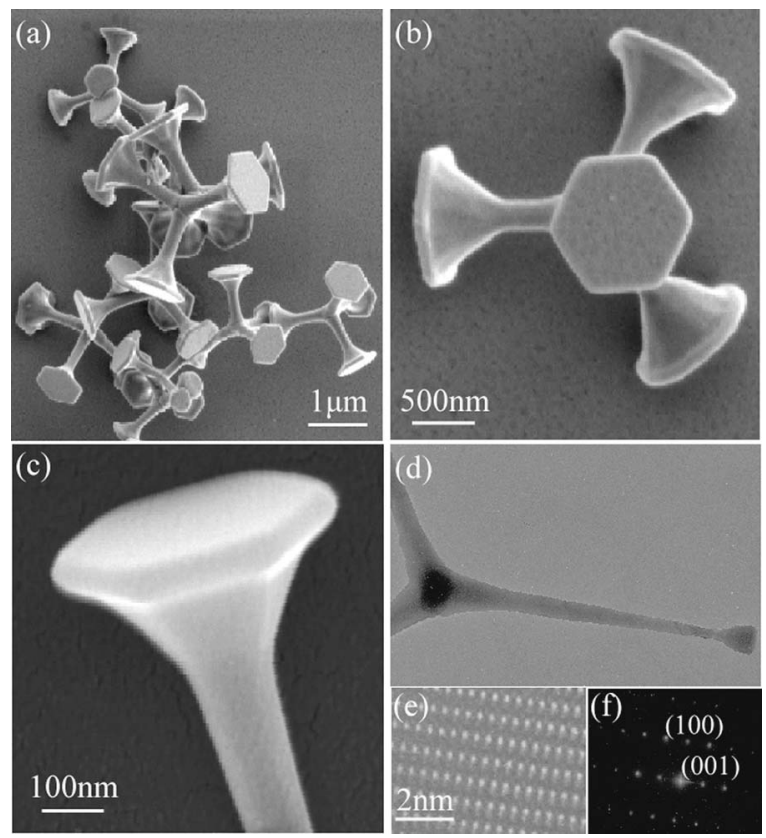

FIG. 2. (a) Low magnification SEM image of $\mathrm{ZnO}$ nanotetrapods with hexagonal crown. [(b) and (c)] High magnification SEM images of the nanostructured $\mathrm{ZnO}$. (d) TEM image of the $\mathrm{ZnO}$ nanotetrapods. (e) HR-TEM

image of the as-grown product. (f) The corresponding SAED pattern.

Downloaded 16 May 2006 to 158.132.12.80. Redistribution subject

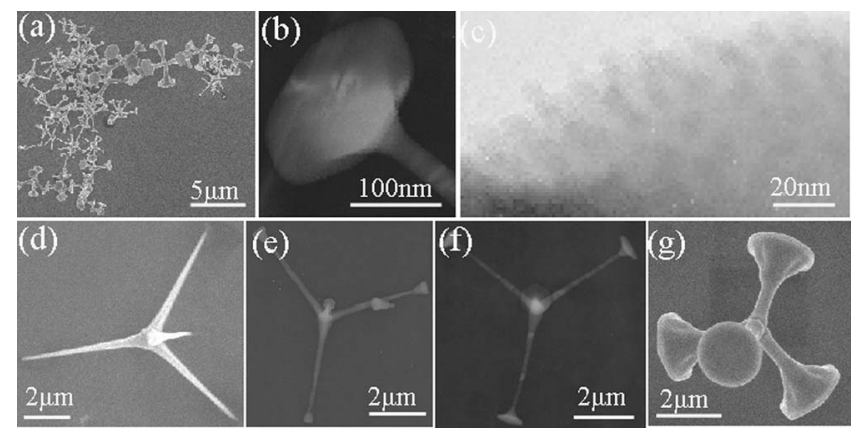

FIG. 3. (a) SEM image showing the nanotetrapods at different stages. [(b) and (c)] HR-TEM images showing the growth way of the nanotetrapods with hexagonal crown. [(d)-(g)] SEM and TEM images showing the formation process of the $\mathrm{ZnO}$ nanotetrapods.

that different nanotetrapods with crown were in different sizes (both in the leg and the crown) which indicates that they were in different stages of the growth process. There exists nanotetrapods with peaked leg, hexagonal bugs, little hexagonal crown, large hexagonal crown, and round crown. We can presume that the as-grown products may originate from the nanotetrapods with peaked leg. If conditions do not change, they may be grown along the same direction with long peaked leg. Actually, we found many as-said structured nanotetrapods in other regions of the boat. But when the condition changes, the growth direction will change also. Apparently, when the $\mathrm{O}_{2}$ is just turned on, the nanotetrapods will grow along the same direction because the $\mathrm{O}_{2}$ is enough to feed the $\mathrm{Zn}$ atoms to form $\mathrm{ZnO}$. However, because the partial pressure of the oxygen is very low, the oxygen will consume quickly in a short period in some region of the boat where the growth of $\mathrm{ZnO}$ will stop, and $\mathrm{Zn}$ atoms (which will further be oxidized to $\mathrm{ZnO}$ immediately again when $\mathrm{O}_{2}$ is available) will gather around the tip in which the surface energy is high. Although, the Zn crystal is also a hexagonal structure, there are some differences between the Zn crystal unit and that of $\mathrm{ZnO}$. The marriage of the change of atmosphere and the gather of $\mathrm{Zn}$ atoms may cause the growth direction to change and create hexagonal crown. We speculate that the nanotetrapods $\mathrm{ZnO}$ with hexagonal crown may grow up into nanotetrapods with round crown in the end.

Figures $3(\mathrm{~d})-3(\mathrm{~g})$ show the growth processes of the $\mathrm{ZnO}$ nanotetrapods with hexagonal crown and the $\mathrm{ZnO}$ nanotetrapods with round crown. Figures 3(b) and 3(c) show the formation way of the crown. Intuitively, we may think they will expand along a crystal face step by step or layer by layer as reported in other literature, ${ }^{13}$ but it is interesting to note that there are a large number of regular protuberances sticking out from the edge around the hexagonal crown, which may further support the conclusion as mentioned above that the formation of the crown may be due to the zinc atoms gathering along the other direction.

The optical properties of the synthesized samples have been studied by using Raman and photoluminescence measurements which were performed at room temperature. According to the group theory, near the Brillouin zone, there are eight different optic modes: $A_{1}, 2 B_{2}, E_{1}$, and $2 E_{2}$, among which $A_{1}, E_{1}$, and $2 E_{2}$ modes are Raman active. ${ }^{14}$ Figure 4 shows the typical Raman scattering of the as-fabricated sample. We observe two dominated and strong intensity peaks at $\sim 99.88$ and $\sim 438.22 \mathrm{~cm}^{-1}$ which are attributed to the low and high- $E_{2}$ mode of nonpolar optical phonons, re-
o AlP license or copyright, see http://apl.aip.org/apl/copyright.jsp 


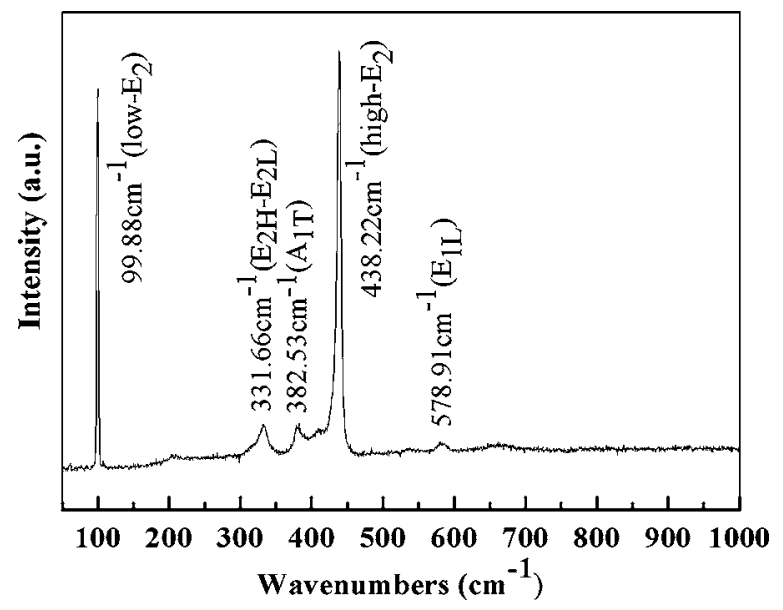

FIG. 4. Raman scattering spectrum acquired from the $\mathrm{ZnO}$ nanotetrapods with hexagonal crown at room temperature.

spectively, and indicating that the as-grown products are excellent single crystal with hexagonal wurtzite structure. ${ }^{15}$ Two very short peaks at $\sim 331.6$ and $382.53 \mathrm{~cm}^{-1}$ correspond to $E_{2 H^{-}} E_{2 L}$ (multiphonons) and $A_{1 T}$ modes, respectively. The short and suppressed peak at $\sim 578.91 \mathrm{~nm}$ is assigned to $E_{1 L}$ mode which is due to the impurities and structural defects (oxygen vacancies and $\mathrm{Zn}$ interstitials ${ }^{16}$ ) of the samples.

Photoluminescence spectra of the $\mathrm{ZnO}$ nanotetrapods with hexagonal crown were measured at room temperature using Xe lamp as the excitation sources. From Fig. 5(a), we observe that the near-band-edge (NBE) ultraviolet (UV) peak at $\sim 393 \mathrm{~nm}(3.37 \mathrm{eV})$ is considerably weak while the green emission at $\sim 511 \mathrm{~nm}(2.43 \mathrm{eV})$ is very strong. It is generally accepted that the green emission is attributed to the single ionized oxygen vacancy in the $\mathrm{ZnO}$, and the emission results from the radiative recombination of a photogenerated hole with an electron occupying the oxygen vacancy, while the UV emission can be explained by the NBE emission of the wide band gap $\mathrm{ZnO}$. So the surface state of the as-made $\mathrm{ZnO}$ determines the green emission peak. In order to prove the result, we annealed the as-deposited products under oxygen at $600{ }^{\circ} \mathrm{C}$ for $30 \mathrm{~min}$. As shown in Fig. 5(b), it is noted that the peak at $\sim 511 \mathrm{~nm}$ becomes very weak after annealing, hereby, we can infer that the green emission is due to the

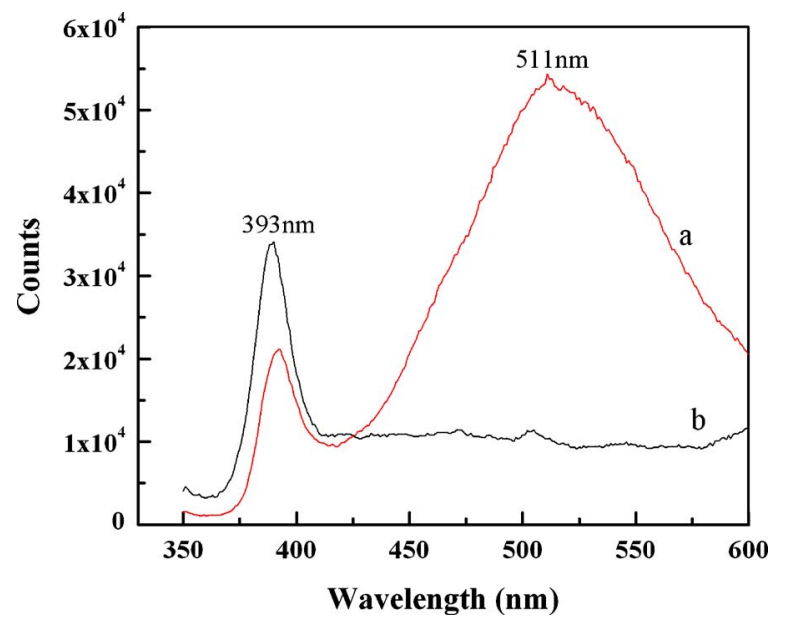

FIG. 5. (Color online) Room temperature photoluminescence spectrum for the $\mathrm{ZnO}$ nanotetrapods: (a) as-synthesized $\mathrm{ZnO}$ nanotetrapods; (b) annealed under oxygen at $600{ }^{\circ} \mathrm{C}$ for $30 \mathrm{~min}$. oxygen vacancies of the as-fabricated materials. As it is well known, the atmosphere and the configuration are important factors for the surface state of the synthesized materials, which means that both the partial pressure of oxygen in the tube and the shape of the samples play crucial roles in the green emission. Because the products have high surface-tovolume ratios for their slender legs and thin hexagonal crown with large surface area, and especially, they have been synthesized at very low oxygen partial pressure, there may exist more oxygen vacancies than other nanotetrapods. Therefore, the green emission peak at $511 \mathrm{~nm}$ is extraordinary strong and quite wide. It is reported that the higher density of oxygen vacancies the nanostructured materials have, the higher electrical conductivity they possess, ${ }^{15}$ therefore, the assynthesized materials are promising candidates as electron nanoconductors in nano-optoelectronic devices. Also, the crystalline (impurity and structure defects such as oxygen vacancy) and the structure (shape, diameter, etc.) of the synthesized $\mathrm{ZnO}$ play key roles in the enhancement of $\mathrm{NBE}$ emission.

In summary, $\mathrm{ZnO}$ nanotetrapods with hexagonal crown have been fabricated via vapor transport process at a low temperature in argon atmosphere with a small amount of oxygen. The growth mechanism has been discussed in detail. XRD, SEM, HR-TEM, and SAED analyses show that the as-produced samples are single crystal with uniform legs and large hexagonal crown. XRD pattern, Raman measurement, and photoluminescence spectra of the nanotetrapods confirm that the as-grown products were wurtzite structured $\mathrm{ZnO}$ with many oxygen vacancies. The products may have potential applications for electron nanoconductors in multichannel optoelectronic devices.

This work was supported by the Distinguished Young Scholar Fund of National Natural Science Foundation of China (NSFC, Grant No. 10225209), key project from Chinese Academy of Sciences (Grant No. KJCX-SW-L2) and NSFC project (Grant No. 90305020).

${ }^{1}$ Z. W. Pan, Z. R. Dai, and Z. L. Wang, Science 291, 1947 (2001).

${ }^{2}$ Y. C. Kong, D. P. Yu, B. Zhang, W. Fang, and S. Q. Feng, Appl. Phys. Lett. 78, 407 (2001).

${ }^{3}$ O. G. Schmidt and K. Eberl, Nature (London) 410, 168 (2001).

${ }^{4}$ M. S. Gudiksen, L. J. Lauhon, J. Wang, D. C. Smith, and C. M. Lieber, Nature (London) 415, 617 (2002).

${ }^{5}$ X. D. Wang, C. J. Summers, and Z. L. Wang, Nano Lett. 4, 423 (2004). ${ }^{6}$ P. D. Yang, H. Q. Yan, S. Mao, R. Russo, J. Johnson, R. Saykally, N. Morris, J. Pham, R. R. He, and H. J. Choi, Adv. Funct. Mater. 12, 323 (2002).

${ }^{7}$ Q. Wan and T. H. Wang, Appl. Phys. Lett. 87, 083105 (2005).

${ }^{8}$ J. O. Hu, Q. Li, X. M. Meng, and C. S. Lee, Chem. Mater. 15, 306 (2003).

${ }^{9}$ J. J. Lao, Y. Huang, D. Z. Wang, and Z. F. Ren, Nano Lett. 3, 235 (2003).

${ }^{10}$ J. Q. Hu, Y. Bando, J. H. Zhan, Y. B. Li, and T. Sekiguchi, Appl. Phys. Lett. 83, 4414 (2003).

${ }^{11}$ P. X. Gao, Y. Ding, W. J. Mai, W. L. Hughes, C. S. Lao, and Z. L. Wang, Science 309, 1702 (2005).

${ }^{12}$ R. C. Wang, C. P. Liu, J. L. Huang, and S. J. Chen, Appl. Phys. Lett. 86, 251104 (2005).

${ }^{13}$ D. F. Liu, Y. J. Xiang, Z. X. Zhang, J. X. Wang, Y. Gao, L. Song, L. F. Liu, X. Y. Dou, X. W. Zhao, S. D. Luo, C. Y. Wang, W. Y. Zhou, G. Wang, and S. S. Xie, Nanotechnology 16, 2665 (2005).

${ }^{14}$ J. M. Calleja and M. Cardona, Phys. Rev. B 16, 3753 (1977).

${ }^{15}$ Y. J. Xing, Z. H. Xi, Z. Q. Xue, X. D. Zhang, J. H. Song, R. M. Wang, J. Xu, Y. Song, S. L. Zhang, and D. P. Yu, Appl. Phys. Lett. 83, 1689 (2003).

${ }^{16}$ K. Vanheusden, C. H. Seager, W. L. Warren, D. R. Tallant, and J. A. Voigt, J. Appl. Phys. 79, 7983 (1996). 